\title{
Problems of Minimal Resistance and the Kakeya Problem
}

\author{
Alexander Plakhov*
}

\begin{abstract}
Here we solve the problem posed by Comte and Lachand-Robert in [8]. Take a bounded domain $\Omega \subset \mathbb{R}^{2}$ and a piecewise smooth nonpositive function $u: \bar{\Omega} \rightarrow \mathbb{R}$ vanishing on $\partial \Omega$. Consider a flow of point particles falling vertically down and reflected elastically from the graph of $u$. It is assumed that each particle is reflected no more than once (no multiple reflections are allowed); then the resistance of the graph to the flow is expressed as $R(u ; \Omega)=\frac{1}{|\Omega|} \int_{\Omega}\left(1+|\nabla u(x)|^{2}\right)^{-1} d x$. We need to find $\inf _{\Omega, u} R(u ; \Omega)$. One can easily see that $|\nabla u(x)|<1$ for all regular $x \in \Omega$, and therefore one always has $R(u ; \Omega)>1 / 2$. We prove that the infimum of $R$ is exactly $1 / 2$. This result is somewhat paradoxical, and the proof is inspired by, and partly similar to, the paradoxical solution given by Besicovitch to the Kakeya problem [1].
\end{abstract}

Mathematics subject classifications: 49Q10, 49K30

Key words and phrases: Newton's problem of least resistance, shape optimization, Kakeya problem.

\section{Introduction}

Consider the following simple mechanical model. A solid body translates through a rarefied medium of point particles at rest. When colliding with the body surface, the particles are reflected in the perfectly elastic way. The medium is so rarefied that mutual interaction of particles can be neglected.

A spacecraft moving forward in an interstellar cloud can serve as a prototype for this model. The cloud is homogeneous and extremely rarefied, and

${ }^{*}$ Center for R\&D in Mathematics and Applications, Department of Mathematics, University of Aveiro, Portugal 
has zero absolute temperature. As a result of collisions with the particles, the drag force acting on the body is created. One is normally interested in finding the body, from a prescribed class of bodies, that minimizes the projection of this force on the direction of motion. This projection is usually called the resistance.

It is natural to impose the condition on the body shape stating that no particle collides with the body more than once. (It will hereafter be referred to as single impact condition, or just SIC. Its analytical form will be derived later; see formula (2).) With this condition, the resistance of the body can be written in a comfortable analytic form. Namely, in a reference system connected with the moving body one observes a parallel flow of particles incident on the body at rest. Introduce orthogonal coordinates $x_{1}, x_{2}, z$ so as the flow falls vertically down with the velocity $(0,0,-1)$, and define the function $u: \Omega \rightarrow \mathbb{R}$, whose graph coincides with the upper part of the body. (Here $\Omega \subset \mathbb{R}^{2}$ is the orthogonal projection of the body on the $\left(x_{1}, x_{2}\right)$-plane, and the graph of $u$ is formed by points of collision with the flow particles.) Then the resistance of the body equals $2 \rho|\Omega| R(u ; \Omega)$, where $\rho$ is the density of the flow, $|\Omega|$ is the area of $\Omega$, and

$$
R(u ; \Omega)=\frac{1}{|\Omega|} \int_{\Omega} \frac{d x}{1+|\nabla u(x)|^{2}},
$$

with $x=\left(x_{1}, x_{2}\right)$ being a point of the plane.

A minimization problem in this setting was first studied by Newton in his Principia [14]. He looked for the body of least resistance in the class of convex axisymmetric bodies with fixed length and width. In terms of functions, the problem (with the length $l$ and width $2 w$ ) reduces to minimizing $R(u ; \Omega)(1)$ in the class of radial concave functions $u: \Omega \rightarrow[0, l]$, where $\Omega$ is the circle with radius $w$. The optimal body with the height equal to the width $(l=2 w)$ is displayed in Fig. 1.

This study gave rise to a series of interesting variational problems that have been intensively studied in the last two decades (see, e.g., [2, 3, 4, 5, 6, 7, $8,11,12,13])$. Let us mention the important problem of minimal resistance in the class of bodies with fixed length $l$ and fixed maximal cross section $\Omega$ and satisfying the single impact condition. (No conditions of convexity or axial symmetry are imposed.) This problem reduces to minimizing $R(u ; \Omega)$ in the class of piecewise smooth functions $u: \Omega \rightarrow[0, l]$ satisfying SIC. This problem was first stated in 1995 in the paper [4] by Buttazzo, Ferone, and Kawohl and further discussed in $[6,7,11]$, and remained open since then.

A very interesting problem was proposed in 2002 by Comte and LachandRobert [8]: minimize $R(u ; \Omega)$ over all bounded domains $\Omega \subset \mathbb{R}^{2}$ and piecewise 


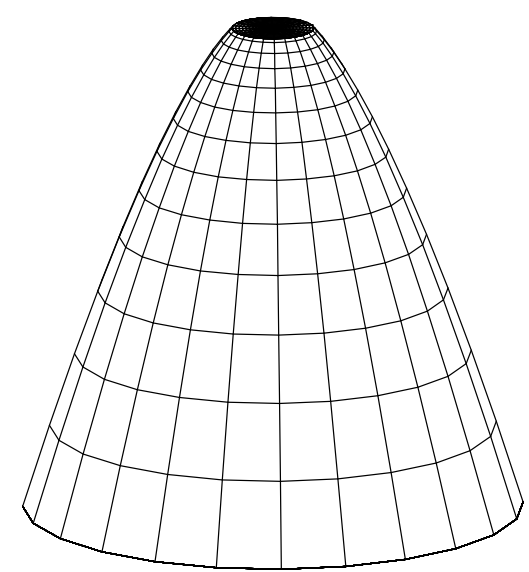

Figure 1: The solution of Newton's problem in the case when the height is equal to the width.

$C^{1}$ functions $u: \bar{\Omega} \rightarrow \mathbb{R}$ satisfying SIC and such that $\left.u\right\rfloor_{\partial \Omega}=0$ and $u(x)<0$ for all $x \in \Omega$.

This problem admits the following mechanical interpretation. A spacecraft moves through a rarefied cloud. One wants to make small dimples on the front part of the spacecraft surface so as to diminish its resistance. The problem amounts to optimization of the shape of dimples. Of course, the mechanical assumptions adopted here are oversimplified, especially as concerns perfectly elastic reflections of particles of the cloud from the spacecraft.

Surprisingly, some problems of minimal resistance can be solved using methods borrowed from the Kakeya problem. In this paper we provide the solution of the problem by Comte and Lachand-Robert; the solution of the Buttazzo-Ferone-Kawohl problem is technically more involved and is given in $[16]$.

The Kakeya needle problem (1917) is as follows: find a plane domain of smallest area in which a line segment of unit length can be continuously turned around through $360^{\circ}$. A surprising answer was given by Besicovitch in 1928: there exist domains of arbitrarily small area with this property. An elementary proof of this fact can be found in [1].

Nowadays there is an extensive literature on Kakeya-type problems and their applications in various areas of mathematics (see, e.g., expository articles [17] and [18]). A striking example of such an application in harmonic analysis is the disproof of the ball multiplier conjecture using a Besicovitchlike construction in the paper of Fefferman [10]. Here we use a similar construction to design a sequence of functions minimizing the resistance.

The single impact condition (SIC) was introduced in [4]. It can can be 
stated analytically as follows: for any regular point $x \in \Omega$ and any $t>0$ such that $x-t \nabla u(x) \in \bar{\Omega}$,

$$
\frac{u(x-t \nabla u(x))-u(x)}{t} \leq \frac{1}{2}\left(1-|\nabla u(x)|^{2}\right) .
$$

For the reader's convenience, here we derive this formula.

SIC means that each particle of the vertical flow with the velocity $(0,0,-1)$, after the perfectly elastic reflection from a regular point of the graph of $u$, further moves freely above the graph (it may, however, touch the graph at some points). Let a particle initially move according to

$$
x(\tau)=x \in \Omega, \quad z(\tau)=-\tau, \quad \tau \leq-u(x) .
$$

The particle reflects from $\operatorname{graph}(u)$ at $(x, u(x))$, and its velocity after the reflection is

$$
v^{+}(x)=\frac{\left(-2 \nabla u(x), 1-|\nabla u(x)|^{2}\right)}{1+|\nabla u(x)|^{2}} .
$$

Its motion after the reflection is described by

$$
\begin{gathered}
x(\tau)=x-\frac{2 \nabla u(x)}{1+|\nabla u(x)|^{2}}(\tau+u(x)), \\
z(\tau)=u(x)+\frac{1-|\nabla u(x)|^{2}}{1+|\nabla u(x)|^{2}}(\tau+u(x)), \quad \tau \geq-u(x) .
\end{gathered}
$$

According to SIC, one has

$$
u(x(\tau)) \leq z(\tau) \quad \text { whenever } \tau \geq-u(x) \text { and } x(\tau) \in \bar{\Omega} .
$$

Substituting $x(\tau)(4)$ and $z(\tau)(5)$ in this inequality and changing the variable $t=2(\tau+u(x)) /\left(1+|\nabla u(x)|^{2}\right)$, one comes to formula (2).

In Figure 2 examples of functions satisfying and not satisfying the SIC are given.

A piecewise $C^{1}$ function $u: \bar{\Omega} \rightarrow \mathbb{R}$ satisfying SIC and such that $\left.u\right\rfloor_{\partial \Omega}=0$ and $u(x)<0$ for all $x \in \Omega$ will be called admissible.

Resistance (1) and SIC (2) are invariant under isometries and dilations of the $x$-plane. Namely, consider a bounded domain $\Omega$ and an admissible function $u: \Omega \rightarrow \mathbb{R}$. Let $k>0$ and $f$ be an isometry of the plane. Take $\tilde{\Omega}=f(k \Omega)$, and define the function $\tilde{u}: \overline{\tilde{\Omega}} \rightarrow \mathbb{R}$ by

$$
\tilde{u}(f(k x))=k u(x) \text { for } x \in \bar{\Omega} .
$$

Proposition 1. The function $\tilde{u}$ is admissible and $R(\tilde{u} ; \tilde{\Omega})=R(u ; \Omega)$. 


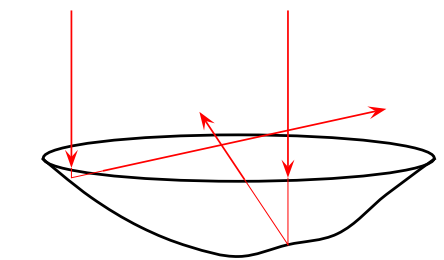

(a)

(b)

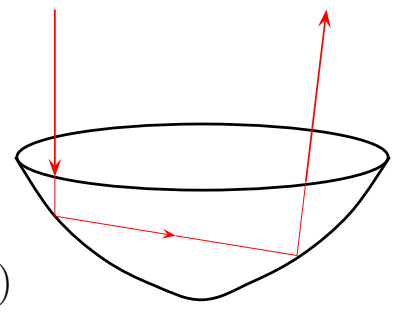

Figure 2: Examples of a function that (a) satisfies and (b) does not satisfy the single impact condition.

The proof is given in the appendix.

Reiterating, the problem of Comte and Lachand-Robert reads as follows.

Problem. Minimize the functional $R(u ; \Omega)$ (1) over all bounded domains $\Omega$ and admissible functions $u: \bar{\Omega} \rightarrow \mathbb{R}$.

Remark 1. The original formulation in [8] concerns the (seemingly more restricted) problem of minimization over bounded domains $\Omega$ tiling the plane. However, as follows from Theorem 2 below, these problems are equivalent.

Two other interesting problems of minimal resistance for bodies satisfying the single impact condition, with and without rotational symmetry, were studied by Comte and Lachand-Robert in [6] and [7].

It is easy to see that for any domain $\Omega, \sup _{u} R(u ; \Omega)=1$, and the supremum is attained, as $n \rightarrow \infty$, at any sequence of functions of the form $\frac{1}{n} u(x)$. On the other hand, for any admissible function $u$ and any regular point $x \in \Omega$ we have

$$
|\nabla u(x)|<1
$$

This can be derived from both geometric considerations and formula (2). Indeed, if $|\nabla u(x)| \geq 1$, the particle reflected from the point $(x, u(x))$ will then move downward (the third component of its velocity will be nonpositive) and therefore will inevitably hit the graph of $u$ once again.

One can also use analytical reasoning: when $x-t \nabla u(x) \in \partial \Omega$, the lefthand side of (2) is positive, and therefore the right-hand side should also be positive.

As a result, one always has $R(u ; \Omega)>1 / 2$, and so, for any $\Omega$

$$
\sup _{u} R(u ; \Omega)=1 \quad \text { and } \quad \inf _{u} R(u ; \Omega) \geq 1 / 2 .
$$




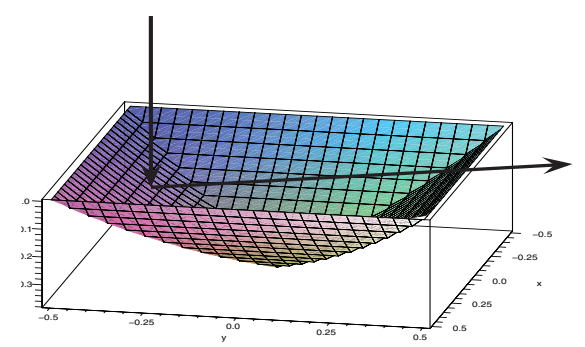

(a)

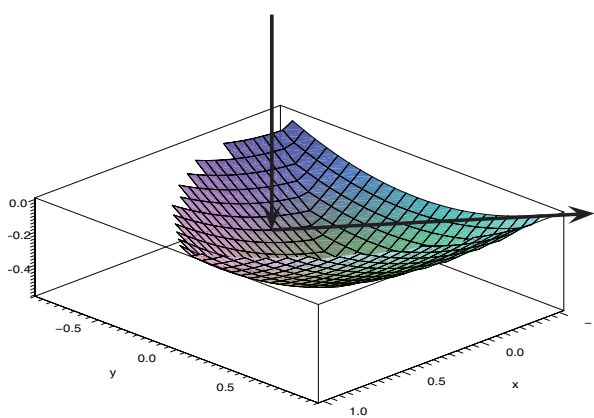

(b)

Figure 3: Graphs of $u^{a}$ and $u^{b}$; a side view. The images are generated by Maple. The trajectory of a single particle of the flow is shown in each case.

Until now it was not even known whether $\inf _{\Omega, u} R(u ; \Omega)$ is equal to or greater than $1 / 2$. It was found in [8] that

$$
R\left(u^{a} ; \Omega^{a}\right) \approx 0.593
$$

for the function $u^{a}\left(x_{1}, x_{2}\right)=\max \left\{\varphi\left(\left|x_{1}\right|+1 / 2\right), \varphi\left(\left|x_{2}\right|+1 / 2\right)\right\}$, with $\varphi(r)=$ $\left(r^{2}-1\right) / 2$ and $\Omega^{a}=(-1 / 2,1 / 2) \times(-1 / 2,1 / 2)$. Additionally, it was shown in $[15]$ that

$$
R\left(u^{b} ; \Omega^{b}\right) \approx 0.581,
$$

where $u^{b}$ and $\Omega^{b}$ are defined as follows. Take an equilateral triangle $A B C$ with unit sides and denote by $r_{A}(x), r_{B}(x), r_{C}(x)$ the distances from $x$ to $A, B, C$; then $u^{b}(x)=\max \left\{\varphi\left(r_{A}(x)\right), \varphi\left(r_{B}(x)\right), \varphi\left(r_{C}(x)\right)\right\}$ and $\Omega^{b}=\{x:$ $\left.r_{A}(x)<1, r_{B}(x)<1, r_{C}(x)<1\right\}$ is a Reuleaux triangle. The images of $u^{a}$ and $u^{b}$ generated by Maple are shown in Figure 3. (The indentation on the boundary of the graph (b) is an artifact of the computer program used.)

Thus, it was first found that $\inf _{u} R(u ; \Omega)<0.594$, and this estimate was then substituted with a better one, $\inf _{u} R(u ; \Omega)<0.582$. The function $u^{a}$ and then the function $u^{b}$ were for some time considered as true minimizers of the Comte-Lachand-Robert problem.

Here we state the main results of this paper.

Theorem 1. $\inf _{u, \Omega} R(u ; \Omega)=1 / 2$.

As a simple corollary of Theorem 1, we get the next result as well.

Theorem 2. For any domain $\Omega$ one has $\inf _{u} R(u ; \Omega)=1 / 2$.

These results are quite paradoxical; they mean that most of the graph of $u$ should be formed by "mirrors" with the angle of inclination close to $45^{\circ}$. 
After reflection from these mirrors, the particles should move a very long way along gently sloping "valleys" below the "zero level," and the total area of these valleys should be close to zero. In the next section we give a solution which, in part, is close to another paradoxical solution given by Besicovitch to the Kakeya problem [1].

\section{Proof of the main results}

The main element of the underlying construction is a triangle divided by a segment into a trapezoid (which is associated with a mirror) and another triangle (associated with a valley); see Fig. 4. One takes a large collection of such elements, in which the mirrors are disjoint and the valleys strongly intersect, so that the area of each mirror is small as compared with the area of the corresponding valley, while the area of the union of valleys is small as compared with the area of the union of mirrors (see Fig. 7).

The domain $\tilde{\Omega}$ is the union of triangles in the collection, and an admissible function $\tilde{u}$ is defined on $\bar{\Omega}$ in such a way that its derivative is close to 1 on the mirrors. A particle reflected by a mirror further moves almost horizontally along the corresponding valley. This is the main idea of the proof of Theorem 1. To prove Theorem 2, one covers a most part of the corresponding domain $\Omega$ by nonintersecting small copies of $\bar{\Omega}$.

The construction of the collection of triangles is close to the constructions used by Cunningham in [9] to design a simply connected solution of the Kakeya problem and by Fefferman in [10] to provide a counterexample to the ball multiplier conjecture. For the reader's convenience, it is described below in detail.

Let us first introduce some notation. Take a triangle $A B C$ on the $x$-plane and draw a segment $M N$ parallel to its base $A C$ with endpoints on the two lateral sides (see Figure 4). The triangle $A B C$ and the segment $M N$ will be called the big triangle and the separating segment, respectively. The open sets $M B N$ and $A M N C$ will be called the small triangle and the trapezoid associated with the big triangle $A B C$, and denoted by the signs $\triangle$ and $\square$. The ratio

$$
\kappa=\frac{\operatorname{dist}(B, M N)}{\max \{|A B|,|B C|\}}
$$

will be called the ratio associated with the big triangle $A B C$, and $\max \{|A B|,|B C|\}$ will be denoted by $r_{0}$. Here of $\operatorname{course} \operatorname{dist}(B, M N)$ means the smallest distance from $B$ to a point of the segment $M N$. In Figure 4, the ratio equals $\kappa=|B H| /|B C|$, where $B H$ is the height of the small triangle, and $r_{0}=|B C|$. 


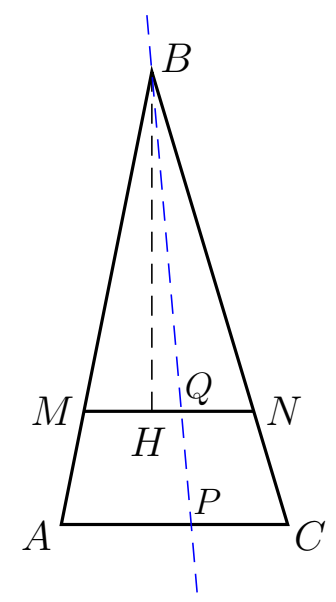

Figure 4: A big triangle.

The values $\kappa$ and $r_{0}$ can also be characterized as follows: the smallest ring centered at $B$ that contains $\square$ has outer radius $r_{0}$ and inner radius $\kappa r_{0}$.

Introduce polar coordinates on the $x$-plane $r=r(x), \theta=\theta(x)$ with the pole at $B$, and define the function $u_{A B C}$ in the closed domain $A B C$ by

$$
u_{A B C}(x)= \begin{cases}\frac{r^{2}(x)-r_{0}^{2}}{2 r_{0}} & \text { if } x \in \square \\ -c & \text { if } x \in \triangle \cup M N \\ 0 & \text { if } x \in A B \cup B C \cup C A\end{cases}
$$

The positive constant $c$ is chosen so as

$$
-c \leq \inf _{x \in \square} u_{A B C}(x)
$$

thereby the function $u_{A B C}$ is not uniquely defined. The function is negative in the interior of the triangle $A B C$ and is zero on its sides.

The graph of the restriction of $u_{A B C}$ on $\square$ is a piece of a circular paraboloid with vertical axis and with focus at $(B, 0) \in \mathbb{R}^{3}$. This means that a particle of the flow reflected from this piece of paraboloid will then move along a ray through the focus. Thus, the third coordinate of the reflected particle will gradually increase, and therefore no further reflections will happen (the trajectory touches the graph of $u_{A B C}$ only at the point $(B, 0)$ ). Further, a particle hitting the graph at a point corresponding to the small triangle $M B N$ (where $u_{A B C}$ is constant) is reflected back in the vertical direction and does not make reflections anymore. Thus, the function $u_{A B C}$ satisfies the SIC.

This can also be checked in a purely analytical way. Indeed, if $x \in \square$, then the vector $\nabla u_{A B C}(x)$ is proportional to $\overrightarrow{B x}$; further, $\left|\nabla u_{A B C}(x)\right|^{2}=r^{2}(x) / r_{0}^{2}$, 


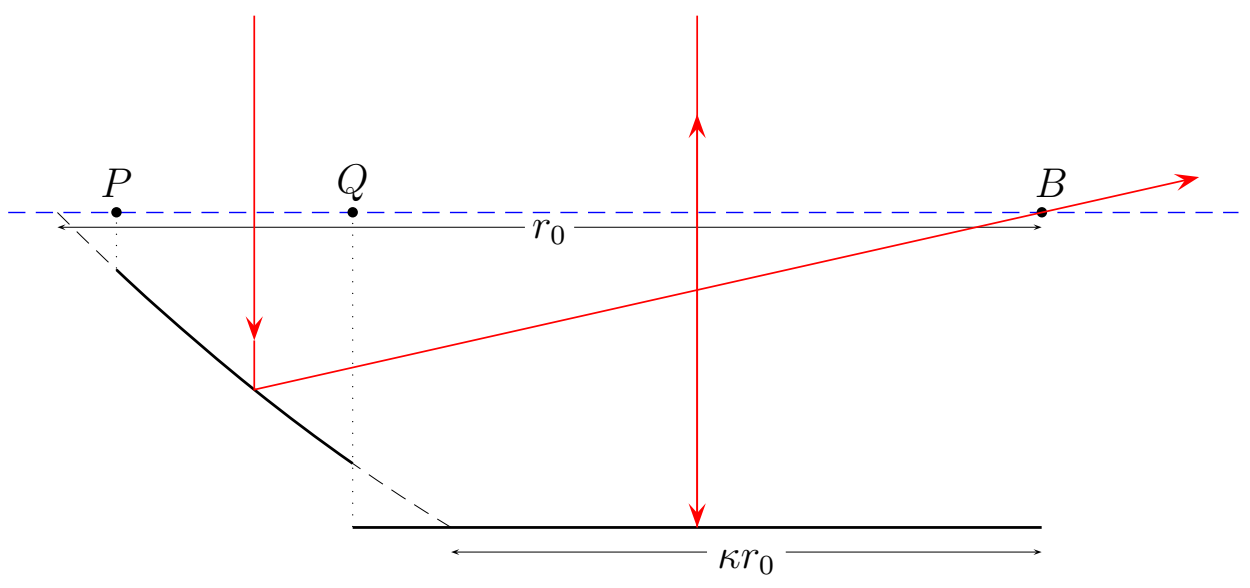

Figure 5: A vertical section of the graph of $u_{A B C}$.

and the point $x-t \nabla u_{A B C}(x)$ lies on the segment $[B, x]$. One can easily see that $u\left(x-t \nabla u_{A B C}(x)\right)<u_{A B C}(x)$ (here one should consider the two cases where $x \in \square$ and $x \in \triangle$ ), and therefore the left-hand side of (2) is negative while the right-hand side is positive. If, otherwise, $x \in \triangle$, then $\nabla u_{A B C}(x)=0$, and therefore the left-hand side of (2) equals zero and the right-hand side equals $1 / 2$.

A section of the graph of $u_{A B C}$ by the vertical plane through a horizontal straight line containing $B$ (the line $P B$ in Figure 4) is shown in Figure 5. The section is formed by an arc of parabola and a horizontal segment. Each particle of the flow that initially belongs to the plane of section, after reflection from the arc or the segment will also belong to this plane. A particle that hits the arc of the parabola will then pass through $B$ (see Figure 5 ). One can also see that $|P B|<r_{0}$ and $|Q B|>\kappa r_{0}$. In Figure 5 the smallest possible value of the constant, $c=-\inf _{x \in \triangle} u_{A B C}(x)$, has been chosen.

One can now estimate the resistance associated with the trapezoid,

$$
\left.R\left(u_{A B C}\right\rfloor_{\square} ; \square\right)=\frac{1}{|\square|} \int_{\square} \frac{d x}{1+\frac{r^{2}(x)}{r_{0}^{2}}} .
$$

Taking into account that $r(x) / r_{0} \geq \kappa$, one obtains that

$$
\left.R\left(u_{A B C}\right\rfloor_{\square} ; \square\right) \leq \frac{1}{1+\kappa^{2}}
$$

On the other hand, the resistance of the small triangle equals 1.

Intuitively, if $\kappa$ is close to 1 , the slope of motion of reflected particles will be small, and therefore the resistance will be close to $1 / 2$. However, in this 
case the relative area of the small triangle (and therefore the resistance of the big triangle $A B C$ ) will be close to 1 . The idea of the proof is to take a large collection of big triangles such that the associated small triangles effectively overlap, and so the relative area of their union is small.

For each natural $n$ we take a family of $2^{n}$ big triangles enumerated by $k=1,2,3, \ldots, 2^{n}$. Let $\kappa_{k}^{n}$ be the associated ratios, let $\triangle_{k}^{n}$ and $\square_{k}^{n}$ be the corresponding small triangles and trapezoids, and denote

$$
\triangle^{n}=\bigcup_{k=1}^{2^{n}} \triangle_{k}^{n} \quad \text { and } \quad \square^{n}=\bigcup_{k=1}^{2^{n}} \square_{k}^{n} .
$$

Proposition 2. There exist families of triangles $\triangle_{k}^{n}, n=1,2, \ldots, k=$ $1, \ldots, 2^{n}$, satisfying the following conditions:

(i) for each $n$, the sets $\square_{1}^{n}, \square_{2}^{n}, \ldots, \square_{2^{n}}^{n}, \triangle^{n}$ are mutually disjoint;

(ii) $\lim _{n \rightarrow \infty}\left|\triangle^{n}\right| /\left|\triangle^{n}\right|=0$;

(iii) there exists a sequence $a_{n}>0$ converging to zero as $n \rightarrow \infty$ such that $\kappa_{k}^{n} \geq 1-a_{n}$.

This proposition is a key point of the proof. At the first glance it looks paradoxical: according to (iii), the area of each trapezoid $\triangle_{k}^{n}$ is much smaller than the area of $\triangle_{k}^{n}$. On the other hand, (ii) implies that the small triangles $\triangle_{k}^{n}, k=1, \ldots, 2^{n}$, strongly overlap, so that the area of their union is much smaller than the area of the union of trapezoids $\square_{k}^{n}$.

Proof. First we define the procedure of $\delta$-doubling. Take a big triangle $A B C$ with the separating segment $M N$, and let $T$ be the midpoint of $M N$ (see Figure 6). Define $|M N|=a$, and let the height of the small triangle $M B N$ be $h$ and the height of the trapezoid $A M N C$ be $d$. Extend the sides $M B$ and $N B$ beyond the point $B$ to obtain the segments $M M^{\prime}$ and $N N^{\prime}$, with

$$
\left|B M^{\prime}\right|=\delta|B M| \quad \text { and } \quad\left|B N^{\prime}\right|=\delta|B N| \text {. }
$$

Let the straight lines $M^{\prime} T$ and $N^{\prime} T$ intersect the segment $A C$ at the points $C^{\prime}$ and $A^{\prime}$, respectively; $N^{\prime} T$ intersects $M B$ at the point $R$, and $M^{\prime} T$ intersects $N B$ at the point $S$. The procedure of $\delta$-doubling applied to $A B C$ results in the two new big triangles $A M^{\prime} C^{\prime}$ and $A^{\prime} N^{\prime} C$; their separating lines $M T$ and $T N$ have the length $a / 2$ each. The new small triangles $M M^{\prime} T$ and $N N^{\prime} T$ have the same height $(1+\delta) h$. The two obtained trapezoids do not intersect and belong to the original trapezoid, and the area of their union is greater than $a d$. 


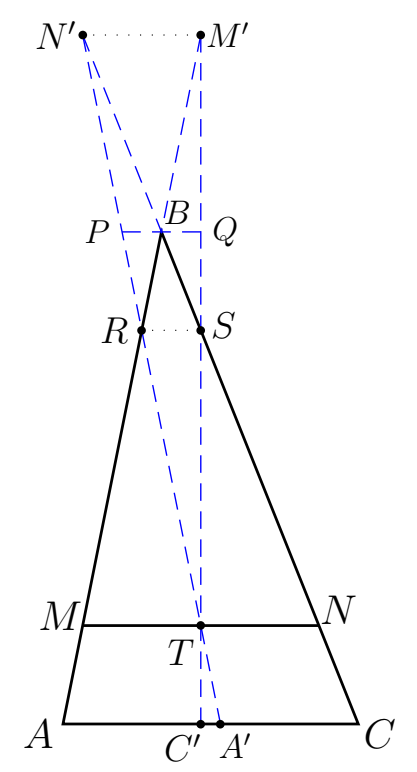

Figure 6: The procedure of doubling: The triangle $A B C$ is replaced with the triangles $A M^{\prime} C^{\prime}$ and $A^{\prime} N^{\prime} C$.

Draw the line through $B$ parallel to $M N$, and denote by $P$ and $Q$ the points of its intersection with $N^{\prime} T$ and $M^{\prime} T$. The triangles $T N^{\prime} N$ and $P N^{\prime} B$ are similar, with the ratio $\delta /(1+\delta)$; therefore

$$
|P B|=\frac{\delta}{1+\delta}|T N|=\frac{\delta}{1+\delta}|M T|
$$

Thus, the triangles $R P B$ and $R M T$ are similar with the same ratio; in particular, we have $|R B|=\delta|M R| /(1+\delta)$, and hence

$$
|R B|=\frac{\delta}{1+2 \delta}|M B|
$$

Consider the triangles $M B N$ and $R B N^{\prime}$. Relation (8) gives the ratio of their sides $R B$ and $M B$, and the ratio of their heights dropped to these sides equals $\delta$. Therefore the area of the triangle $R B N^{\prime}$ equals

$$
\left|R B N^{\prime}\right|=\frac{\delta^{2}}{1+2 \delta}|M B N|=\frac{\delta^{2}}{1+2 \delta} \cdot \frac{a h}{2} .
$$

The area of the triangle $S B M^{\prime}$ is the same. Thus, the increase of the total area as a result of doubling is less than $\delta^{2} a h$.

Let us now apply the procedure of doubling several times. Initially one has the triangle $A B C$, and at the $m$ th step $(m \geq 1)$ one applies the procedure 
of $\delta_{m}$-doubling to each of $2^{m-1}$ triangles obtained at the previous step. Thus, the separating lines of the triangles at the $m$ th step have the length $2^{-m} a$ each, and their union is the segment $M N$. The height of each such triangle equals

$$
h_{m}=\left(1+\delta_{m}\right) \cdots\left(1+\delta_{1}\right) h,
$$

and its area equals $2^{-m-1} a \cdot h_{m}$.

Let $S_{m}$ be the area of the union of triangles at the $m$ th step. The increase of the area at the $(m+1)$ th step is smaller than $2^{m} \cdot \delta_{m+1}^{2} 2^{-m} a h_{m}$; that is,

$$
S_{m+1}<S_{m}+\delta_{m+1}^{2} a h_{m}
$$

Take $h_{m}=m+1$ (and in particular, $h=h_{0}=1$ ); then we have $\delta_{m}=1 / m$ and $S_{0}=a / 2$, and by $(9)$,

$$
S_{m+1}<S_{m}+\frac{a}{m+1}
$$

One easily concludes that $S_{m}<a(\ln m+3 / 2)$ for $m \geq 1$.

In Figure 7 the initial triangle and the triangles obtained in steps $1-3$ of the doubling procedure are shown.

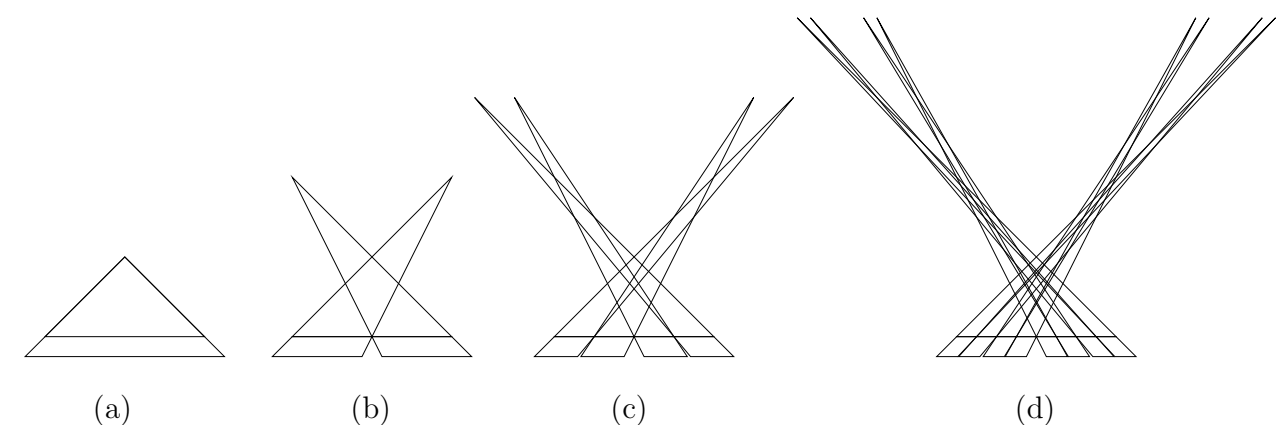

Figure 7: The original triangle (a), two triangles obtained at the first step (b), four triangles obtained at the second step (c), and eight triangles obtained at the third step $(\mathrm{d})$.

For all $m$, the trapezoids of the $m$ th step are disjoint. Indeed, let this be true at the $m$ th step. Two trapezoids obtained when doubling a triangle of the $m$ th step are disjoint and are contained in the trapezoid associated with the original triangle; therefore they do not intersect any other trapezoid obtained at the $(m+1)$ th step. The trapezoids also do not intersect the small triangles, since they lie on the opposite sides of the line $M N$. The total area of the trapezoids is greater than $a d$. 
Fix $n$ and take $d=\sqrt{n}$. Let $\triangle_{k}^{n}$ and $\square_{k}^{n}, k=1, \ldots, 2^{n}$, be the triangles and trapezoids of the $n$th step. We have already verified that Proposition $2(\mathrm{i})$ is true. Further,

$$
\left|\triangle^{n}\right|=\left|\bigcup_{k=1}^{2^{n}} \triangle_{k}^{n}\right|=S_{n}<a\left(\ln n+\frac{3}{2}\right)
$$

and the total area of the trapezoids is

$$
\left|\square^{n}\right|=\left|\bigcup_{k=1}^{2^{n}} \square_{k}^{n}\right|>a \sqrt{n}
$$

therefore (ii) is also fulfilled.

Now let $A_{k} B C_{k}$ be the $k$ th big triangle $\left(1 \leq k \leq 2^{n}\right)$, and $M_{k} N_{k}$ be its separating line. Assume without loss of generality that $\left|A_{k} B\right| \geq\left|C_{k} B\right|$. Using that

$$
\begin{gathered}
\frac{\left|M_{k} B\right|}{\left|A_{k} B\right|}=\frac{h_{n}}{h_{n}+d}=\frac{n+1}{n+1+\sqrt{n}}, \\
\left|M_{k} N_{k}\right|=2^{-n} a, \text { and }\left|A_{k} B\right|>n+1+\sqrt{n}, \text { one obtains } \\
\kappa_{k}^{n}=\frac{\operatorname{dist}\left(B, M_{k} N_{k}\right)}{\left|A_{k} B\right|} \geq \frac{\left|M_{k} B\right|-\left|M_{k} N_{k}\right|}{\left|A_{k} B\right|} \geq \frac{n+1}{n+1+\sqrt{n}}-\frac{2^{-n} a}{n+1+\sqrt{n}},
\end{gathered}
$$

so (iii) is also satisfied.

Now we use relation (7) to define the function $u_{k}^{n}$ in each big triangle, choosing the constant $c=c_{n}$ to be the same for all $k$. Let $\Omega_{n}=\triangle^{n} \cup \square^{n}$. Using (i), we define the function $u_{n}$ on $\bar{\Omega}_{n}$ so that its restriction on each big triangle of the $n$th step coincides with the corresponding function $u_{k}^{n}$. Using (ii) and (iii), we now obtain the estimates for the resistance of $u_{n}$ :

$$
R\left(u_{n} ; \Omega_{n}\right) \leq \frac{\left|\triangle^{n}\right|}{\left|\triangle^{n}\right|+\left|\triangle^{n}\right|}\left(\frac{1}{1+\left(1-a_{n}\right)^{2}}\right)+\frac{\left|\triangle^{n}\right|}{\left|\triangle^{n}\right|+\left|\triangle^{n}\right|} \rightarrow \frac{1}{2} \quad \text { as } n \rightarrow \infty .
$$

Thus, $\inf _{u, \Omega} R(u ; \Omega)=1 / 2$, and Theorem 1 is proved.

Remark 2. The sequence of domains $\Omega_{n}$ does not converge in any reasonable sense. On the contrary, the domains become more and more complicated as $n$ grows. It may seem disturbing that the size of $\Omega_{n}$ goes to infinity. However, applying the scaling transformation $\tilde{\Omega}_{n}=\frac{1}{n} \Omega_{n}, \tilde{u}_{n}(x)=\frac{1}{n} u_{n}(n x)$, one comes to the sequence of domains $\tilde{\Omega}_{n}$ with bounded diameter, and by Proposition 1 one has $R\left(\tilde{\Omega}_{n} ; \tilde{u}_{n}\right)=R\left(\Omega_{n} ; u_{n}\right) \rightarrow 1 / 2$ as $n \rightarrow \infty$. 
Let us now prove Theorem 2 .

We say that $\Omega^{i}$ is a copy of $\Omega$ if there exist a real value $k_{i}>0$ and an isometry $f_{i}$ of the plane such that $f_{i}\left(k_{i} \Omega\right)=\Omega^{i}$.

Proposition 3. Let $\varepsilon>0$. Then for any two bounded domains $\Omega$ and $\tilde{\Omega}$ there exists a finite family $\left\{\Omega^{i}\right\}$ of mutually nonintersecting copies of $\Omega$, all contained in $\tilde{\Omega}$, such that $\left|\tilde{\Omega} \backslash\left(\cup_{i} \Omega^{i}\right)\right|<\varepsilon$.

Proof. Take a square $\left|x_{1}\right|<M,\left|x_{2}\right|<M$ containing $\Omega$ and fix $\delta=|\Omega| / M^{2}$. Obviously, $0<\delta<1$, and any square $Q$ on the plane contains a copy of $\Omega$ that occupies the area $\delta|Q|$. Further, take a square lattice $x_{1}=a m, x_{2}=a n, a>$ $0, m, n \in \mathbb{Z}$, choosing a so small that the squares $Q^{i}$ of the lattice contained in the domain $\tilde{\Omega}$ occupy more than one half of its area, $\left|\cup_{i} Q^{i}\right|>\frac{1}{2}|\tilde{\Omega}|$. For each square $Q^{i}$ take a copy $\Omega^{(i 0)}$ of $\Omega$ contained in $Q^{i}$ and such that $\left|\Omega^{(i 0)}\right|=\delta\left|Q^{i}\right|$. Thus, we have

$$
\left|\cup_{i} \Omega^{(i 0)}\right|>\frac{\delta}{2}|\tilde{\Omega}|
$$

Next we inductively define the sequence of domains $\tilde{\Omega}^{j}, j=0,1, \ldots, j_{0}$, and finite families $\Omega^{(i j)}, j=1, \ldots, j_{0}-1$, of copies of $\Omega$ such that for all $j$ the domains of the family $\left\{\Omega^{(i j)}\right\}_{i}$ are mutually disjoint,

$$
\left|\cup_{i} \Omega^{(i j)}\right|>\frac{\delta}{2}\left|\tilde{\Omega}^{j}\right|, \quad \tilde{\Omega}^{0}=\tilde{\Omega}, \quad \tilde{\Omega}^{j+1}=\tilde{\Omega}^{j} \backslash\left(\cup_{i} \Omega^{(i j)}\right) \quad \text { for } 0 \leq j \leq j_{0}-1,
$$

and $(1-\delta / 2)^{j_{0}}<\varepsilon$. We have $\left|\tilde{\Omega}^{j}\right|<(1-\delta / 2)^{j}|\tilde{\Omega}|$, and therefore,

$$
\left|\tilde{\Omega} \backslash\left(\cup_{i, j} \Omega^{(i j)}\right)\right|=\left|\tilde{\Omega}^{j_{0}}\right|<\varepsilon .
$$

Proposition 4. Let $\varepsilon>0$. Then for any pair of bounded domains $\Omega, \tilde{\Omega}$ and any admissible function $u: \bar{\Omega} \rightarrow \mathbb{R}$ there exists an admissible function $\tilde{u}: \bar{\Omega} \rightarrow \mathbb{R}$ such that

$$
R(\tilde{\Omega} ; \tilde{u})<R(\Omega ; u)+\varepsilon
$$

Proof. Take a finite family $\Omega^{i}=f_{i}\left(k_{i} \Omega\right)$ of nonintersecting copies of $\Omega$ contained in $\tilde{\Omega}$ and such that $\left|\tilde{\Omega} \backslash\left(\cup_{i} \Omega^{i}\right)\right|<\varepsilon|\tilde{\Omega}|$. The transformations $f_{i} k_{i}$ induce the functions $u_{i}$ on $\overline{\Omega^{i}}$ by

$$
u_{i}\left(f_{i}\left(k_{i} x\right)\right)=k_{i} u(x) \text { for all } x \in \bar{\Omega} .
$$

By Proposition 1, these functions are admissible and $R\left(u_{i} ; \Omega^{i}\right)=R(u ; \Omega)$. 
Define the admissible function $\tilde{u}$ on $\overline{\widetilde{\Omega}}$ by

$$
\tilde{u}(x)=\left\{\begin{array}{cl}
u_{i}(x) & \text { if } x \in \Omega^{i} \\
-c & \text { if } x \in \tilde{\Omega} \backslash\left(\cup_{i} \Omega^{i}\right) \\
0 & \text { if } x \in \partial \tilde{\Omega}
\end{array}\right.
$$

where $c$ is an arbitrary positive constant. One has

$$
R(\tilde{\Omega} ; \tilde{u})=\sum_{i} \frac{\left|\Omega^{i}\right|}{|\tilde{\Omega}|} R\left(u_{i} ; \Omega^{i}\right)+\frac{\left|\tilde{\Omega} \backslash\left(\cup_{i} \Omega^{i}\right)\right|}{|\tilde{\Omega}|}<R(\Omega ; u)+\varepsilon .
$$

By Theorem 1, there exists a sequence of admissible functions $u_{n}: \bar{\Omega}_{n} \rightarrow$ $\mathbb{R}$ such that $R\left(u_{n} ; \Omega_{n}\right) \rightarrow 1 / 2$. Let $\Omega$ be a bounded domain. By Proposition 4 , for each natural $n$ there exists an admissible function $\tilde{u}_{n}: \bar{\Omega} \rightarrow \mathbb{R}$ such that $R\left(\tilde{u}_{n} ; \Omega\right)<R\left(u_{n} ; \Omega_{n}\right)+1 / n$. This implies that $\inf _{u} R(u ; \Omega)=1 / 2$. Theorem 2 is proved.

\section{Appendix}

Here we prove Proposition 1.

Let $\delta>0$. Consider a finite number of particles (mass points) with the $x$-coordinates at vertices of the square lattice $\delta \mathbb{Z} \times \delta \mathbb{Z}$ falling vertically downward on the graph of $u$. Each particle initially moves according to

$$
x(t)=x=\left(m_{1} \delta, m_{2} \delta\right) \in \Omega \quad\left(m_{1}, m_{2} \in \mathbb{Z}\right), \quad z(t)=-t, \quad t \leq-u(x),
$$

then elastically reflects at $(x, u(x))$, and then moves freely above the graph of $u$ with the velocity $v^{+}(x)$ given by (3). The vertical component of the momentum imparted by the particle to the graph of $u$ equals $-2 \mu /(1+$ $|\nabla u(x)|^{2}$ ), where $\mu$ is the mass of the particles. Taking $\mu=\delta^{2} /|\Omega|$ and summing over all imparted momenta, we find that the vertical component of the total momentum imparted to the graph equals

$$
-\frac{1}{|\Omega|} \sum_{\left(m_{1} \delta, m_{2} \delta\right) \in \Omega \cap(\delta \mathbb{Z} \times \delta \mathbb{Z})} \frac{\delta^{2}}{1+\left|\nabla u\left(m_{1} \delta, m_{2} \delta\right)\right|^{2}} .
$$

This expression is the Riemann integral sum for the integral $-R(u ; \Omega)$ and converges to it as $\delta \rightarrow 0$.

Now successively apply two transformations of $\mathbb{R}^{3}$, the dilation $\varphi:(x, z) \mapsto$ $(k x, k z)$ and the isometry $\psi:(x, z) \mapsto(f(x), z)$. The composition $g=$ 
$\psi \varphi: \mathbb{R}^{3} \rightarrow \mathbb{R}^{3}$ takes the plane domain $\Omega \times\{0\}$ to $\tilde{\Omega} \times\{0\}$ and $\operatorname{graph}(u)$ to $\operatorname{graph}(\tilde{u})$. Moreover, $g$ conjugates the billiard in the complement of $\operatorname{graph}(u)$ and the billiard in the complement of $\operatorname{graph}(\tilde{u})$. In particular, if $(x(t), z(t))$ describes the motion of a particle above graph $(u)$ with the initial data $x(t)=x \in \Omega, z(t)=-t$, then $(\tilde{x}(t), \tilde{z}(t))=g(x(t / k), z(t / k))$ describes the motion above graph $(\tilde{u})$ with the initial data $\tilde{x}(t)=f(k x) \in \tilde{\Omega}, \tilde{z}(t)=-t$. It follows that the motion $(\tilde{x}(t), \tilde{z}(t))$ has a single reflection from $\operatorname{graph}(\tilde{u})$ and then is free. Thus, $\tilde{u}$ satisfies the SIC and is therefore admissible.

Now compare the finite flow of particles incident on graph $(u)$ defined above with the new flow incident on $\operatorname{graph}(\tilde{u})$ with the initial $x$-coordinates in the square lattice $f(k \delta \mathbb{Z} \times k \delta \mathbb{Z})$. The particles of the new flow have mass $\mu$ and initially move according to

$$
\tilde{x}(t)=\tilde{x}=f\left(k m_{1} \delta, k m_{2} \delta\right) \in \tilde{\Omega} \quad\left(m_{1}, m_{2} \in \mathbb{Z}\right), \quad \tilde{z}(t)=-t .
$$

There is a natural one-to-one correspondence between particles in the flows, their masses are equal, and their trajectories are similar. Therefore the momenta imparted by the flows to $\operatorname{graph}(u)$ and $\operatorname{graph}(\tilde{u})$ coincide. Denoting $\tilde{\delta}=k \delta$ and taking into account that $|\tilde{\Omega}|=\delta^{2}|\Omega|$, one obtains that

$$
\begin{aligned}
& \frac{1}{|\Omega|} \sum_{\left(m_{1} \delta, m_{2} \delta\right) \in \Omega \cap(\delta \mathbb{Z} \times \delta \mathbb{Z})} \frac{\delta^{2}}{1+\left|\nabla u\left(m_{1} \delta, m_{2} \delta\right)\right|^{2}} \\
& =\frac{1}{|\tilde{\Omega}|} \sum_{\left(m_{1} \tilde{\delta}, m_{2} \tilde{\delta}\right) \in \tilde{\Omega} \cap f(\tilde{\delta} \mathbb{Z} \times \tilde{\delta} \mathbb{Z})} \frac{\tilde{\delta}^{2}}{1+\left|\nabla \tilde{u}\left(m_{1} \tilde{\delta}, m_{2} \tilde{\delta}\right)\right|^{2}},
\end{aligned}
$$

and, passing to the limit $\delta \rightarrow 0$ on both sides, one gets $R(u ; \Omega)=R(\tilde{u} ; \tilde{\Omega})$.

\section{Acknowledgements}

This work was supported by Portuguese funds through CIDMA (the Center for Research and Development in Mathematics and Applications) and FCT (the Portuguese Foundation for Science and Technology), within the project PEst-OE/MAT/UI4106/2014, as well as by the FCT research project PTDC/MAT/113470/2009.

\section{References}

[1] A.S. Besicovitch, The Kakeya problem, Amer. Math. Monthly, 70 (1963), pp. 697-706. 
[2] F. Brock, V. Ferone, and B. Kawohl, A symmetry problem in the calculus of variations, Calc. Var., 4 (1996), pp. 593-599.

[3] D. Bucur And G. Buttazzo, Variational Methods in Shape Optimization Problems, Birkhäuser Boston, Cambridge, MA, 2005.

[4] G. Buttazzo, V. Ferone, And B. Kawohl, Minimum problems over sets of concave functions and related questions, Math. Nachr., 173 (1995), pp. 71-89.

[5] G. Buttazzo And B. Kawohl, On Newton's problem of minimal resistance, Math. Intell., 15 (1993), pp. 7-12.

[6] M. Comte And T. Lachand-Robert, Newton's problem of the body of minimal resistance under a single-impact assumption, Calc. Var. Partial Differential Equations, 12 (2001), pp. 173-211.

[7] M. Comte And T. Lachand-Robert, Existence of minimizers for Newton's problem of the body of minimal resistance under a single-impact assumption, J. Anal. Math., 83 (2001), pp. 313-335.

[8] M. Comte And T. LACHAnd-Robert, Functions and domains having minimal resistance under a single-impact assumption, SIAM J. Math. Anal., 34 (2002), pp. 101-120.

[9] F. Cunningham JR., The Kakeya problem for simply connected and star-shaped sets, Amer. Math. Monthly, 78 (1971), pp. 114-129.

[10] C. Fefferman, The multiplier problem for the ball, Ann. Math., 94 (1971), pp. 330-336.

[11] B. KaWOHL, Some nonconvex shape optimization problems, in Optimal Shape Design (Tróia, 1998), Lecture Notes in Math. 1740, Springer, Berlin, 2000, pp. 7-46.

[12] T. LACHAnd-Robert And É. OUdet, Minimizing within convex bodies using a convex hull method, SIAM J. Optim., 16 (2005), pp. 368-379.

[13] T. Lachand-Robert And M. A. Peletier, Newton's problem of the body of minimal resistance in the class of convex developable functions, Math. Nachr., 226 (2001), pp. 153-176.

[14] I. Newton, Philosophiae Naturalis Principia Mathematica, Streater, London, 1687. 
[15] A. Plakhov, Comment on "Functions and domains having minimal resistance under a single-impact assumption" [SIAM J. Math. Anal., 34 (2002), pp. 101-120], SIAM J. Math. Anal., 41 (2009), pp. 1721-1724.

[16] A. Plakhov, Newton's problem of minimal resistance under the singleimpact assumption, arXiv:1405.0122 [math.CA].

[17] T. TAO, From rotating needles to stability of waves: emerging connections between combinatorics, analysis, and PDE, Notices of the AMS, 48 (2001), pp. 294-303.

[18] T. WolfF, Recent work connected with the Kakeya problem, Prospects in Mathematics (Princeton, NJ, 1996), Amer. Math. Soc., Providence, RI (1999), pp. 129-162. 